\title{
RECONOCIMIENTO DE TEXTURAS EN IMÁGENES DE PROYECTILES: UN APORTE A LA IDENTIFICACIÓN AUTOMÁTICA DE ARMAS
}

\section{AN IMAGE AMMUNITION TEXTURE RECOGNITION - A CONTRIBUTION TO AN AUTOMATIC WEAPON IDENTIFICATION}

\author{
Jeison Méndez García \\ Ing. Físico, Facultad de Ingenierías Eléctrica, Electrónica, Mecánica y Física, \\ Investigador Grupo de Investigación en Ingeniería Física, \\ Universidad Tecnológica de Pereira, Pereira, Colombia. \\ jmendez@utp.edu.co
}

Jorge Hernando Rivera Piedrahita

Ing. Electrónico, Ms.C., Profesor titular, Facultad de Ingenierías Eléctrica, Electrónica, Mecánica y Física,

Director Grupo de Investigación en Ingeniería Física,

Universidad Tecnológica de Pereira, Pereira, Colombia,

j.rivera@utp.edu.co

José Adalberto Soto Mejía

Físico, Ph.D, Profesor titular, Facultad de Ingeniería Industrial,

Investigador Grupo de Investigación en Ingeniería Física,

Universidad Tecnológica de Pereira, Pereira, Colombia.

jomejia@utp.edu.co

Fecha de recepción: 30 de agosto de 2011

Fecha de aprobación: 29 de mayo de 2012

\section{RESUMEN}

Debido al contacto entre el interior del cañón y el proyectil durante el disparo de un arma de fuego, quedan impresas marcas sobre la superficie del proyectil. Estas marcas son características de cada tipo de arma, y más aún, pueden ser interpretadas como su "huella digital". Tradicionalmente, la identificación de armas con base en el cotejo o comparación de proyectiles, realizadas por los peritos en balística, es una actividad difícil que consume mucho tiempo. La actividad consiste en observar con un macroscopio de comparación, dos proyectiles: el patrón y el incriminado. El objetivo es hallar similitudes entre las marcas que presentan los dos, y determinar si provienen o no de la misma arma. La tarea de identificación demanda el uso de computadoras debido a la cantidad de casos judiciales que implican armas de fuego. En este trabajo se presenta un método para obtener una representación bidimensional de las regiones de interés en las superficies de los proyectiles percutidos, procedimiento que en este trabajo, será llamado "desenvolver" la superficie; después, se usa un método para dotar de características cuantitativas dichas imágenes; por último, el soporte estadístico para mostrar las diferencias entre clases de armas incluidas en el experimento. 
Palabras clave: reconocimiento automático de patrones, análisis estadístico multivariado, cotejo de proyectiles.

\section{ABSTRACT}

Due to a contact between the barrel and projectile as a firearm triggered, markings are printed on surface of a projectile. These marks are typical of each firearm and may be interpreted as a 'firearm fingerprint.' Typically, a firearm identification based on bullet's surface matching is time consuming and consists of two bullets (involved and standard ones), both matched under microscope comparison by experts in order to establish whether they are from the same firearm. The identification task demands computer aids due to a number of court cases involving firearms. This paper offers a method to get a bi-dimension representation of a fired bullet surface along with a method to provide quantitative features of those images. Finally, a statistical support to show differences between analyzed firearms is included.

Keywords: automatic standard recognition, multivariate statistical analysis, bullet matching.

\section{INTRODUCCIÓN}

Identificar las armas de fuego permite unir un crimen con un individuo sospechoso. La necesidad de procesos de identificación más confiables, repetibles y reproducibles se ha convertido en el tema de estudio de muchos investigadores, debido a su importancia en la solución de muerte con armas de fuego.

En la identificación de armas, se usan las marcas impresas en los proyectiles y las vainillas cuando el arma ha sido disparada. Debido al contacto del proyectil con el interior del cañón y de la vainilla con el percutor, la recámara, el extractor y el eyector, aparecen dos tipos de marcas que presentan diferentes características, unas llamadas características de clase que dependen del tipo de arma (compañía fabricante), y permiten identificar parámetros generales tales como la marca, el modelo y el calibre del arma; mientras otras más especiales llamadas características individuales, proporcionan la posibilidad de identificar cada arma en particular, debido a finas imperfecciones en la elaboración de las piezas del arma que entran en contacto con el proyectil y la vainilla. Se puede asumir además, una combinación única de marcas de manera que la probabilidad de encontrar dos conjuntos idénticos de marcas es prácticamente nula [1].

Se pueden distinguir cerca de 30 características de estas marcas, las cuales al combinarse, pueden producir una "huella digital" del arma de fuego. Analizando las características de un conjunto de huellas digitales de armas, es posible identificar el tipo y modelo de arma de fuego y cada arma en particular, de manera tan efectiva como la huella digital humana identifica cada individuo [2]. 
Tradicionalmente, la comparación entre proyectiles disparados con armas de fuego por los técnicos en balística, es una tarea que implica mucho tiempo, debido a que se lleva a cabo, usando sólo la memoria visual y la experiencia del técnico para comparar texturas presentes en la imagen del proyectil objeto de prueba y la imagen del proyectil incriminado, lo cual representa una enorme limitación a la hora de usar este tipo de evidencia en una corte o juzgado.

Por otra parte, la gran cantidad de casos que se investigan y se relacionan con armas de fuego van en aumento, debido al acceso relativamente sencillo de adquirirlas de forma legal o ilegal. Actualmente, el número de casos relacionados con proyectiles disparados por armas de fuego se acerca a los 3.000 casos anuales en la región cafetera colombiana, lo cual sumado al tiempo en la tarea de comparación tradicional, explica el creciente índice de impunidad en casos relacionados con armas de fuego. De esta manera, la identificación de armas de fuego en materia judicial, es una tarea que además demanda fiabilidad, exactitud, y velocidad de procesamiento de la evidencia. Es en este punto, donde cobra importancia el uso de sistemas de procesamiento de imágenes digitales.

Los sistemas automáticos de procesamiento de imágenes son herramientas para agilizar la comparación de evidencia, reducir pronto el número de candidatos. Sin embargo, son los peritos y técnicos en balística quienes deben evaluar el desempeño de los sistemas automáticos de reconocimiento y la emisión de los resultados.

Ahora bien, hace varios años en diferentes países del Mundo, tales como Estados Unidos, Canadá y Australia, los entes encargados de hacer cumplir la ley han llevado a cabo investigaciones para desarrollar dispositivos que capturen las imágenes de proyectiles y vainillas relacionados con investigaciones judiciales, y luego, usando el procesamiento digital de imágenes, dotar de características cuantitativas a los patrones presentes en proyectiles y vainillas disparadas, para facilitar y agilizar la identificación de las armas de fuego.

En la actualidad, existen varios dispositivos en forma comercial y en estado de prueba, para identificar armas de fuego. Los dos sistemas de identificación automática de armas de fuego más reconocidos son fabricados por la compañía IBIS en Montreal-Canadá, y el FBI (Drugfire), en Estados Unidos. Por otra parte, una compañía canadiense, WalshAutomation, desarrolló un sistema comercial llamado Bulletproof, que permite adquirir y almacenar imágenes de proyectiles y vainillas, y luego de manera automática, buscar en una base de datos, estriaciones características de los proyectiles. Sin embargo, es el usuario quien debe igualar las marcas impresas o estrías sobre el proyectil. Esta limitación del sistema con respecto de los proyectiles, ha invalidado su uso. En Australia, la universidad Edith Cowan y el Instituto Australiano de Seguridad y de Tecnología Aplicada han desarrollado el Fireball-Firearm Identification, reconocido en la actualidad como el sistema de identificación de armas más pequeño en el Mundo. El Fireball permite almacenar y recuperar imágenes de la parte posterior de las vainillas y luego de forma interactiva, obtener la posición métrica de la impresión del percutor, la marca del eyector y la marca del extractor. La limitación de este sistema es que la posición y la forma de las impresiones en las imágenes, deben ser trazadas manualmente por el usuario. A pesar de lo anterior, 
el sistema ha estado cuatro años en funcionamiento. Por el momento, sigue sin resolverse el problema de análisis, almacenamiento y adquisición de las imágenes de proyectiles [3,4].

\section{MATERIALES Y MÉTODOS}

A continuación, se resume cada etapa del trabajo realizado. Primero se hizo la construcción de la base de datos a partir de las imágenes de los proyectiles analizados en el trabajo. Después, se hizo una descripción del sistema usado en la adquisición de las imágenes de los proyectiles; luego el pre-procesamiento que fue aplicado a cada imagen, posteriormente la descripción de la naturaleza de las características propuestas como descriptores de las texturas; y por último, se incluyeron los resultados de las pruebas de significancia estadística de las diferencias entre los diferentes tipos de armas, para los diferentes conjuntos de descriptores propuestos.

\subsection{CONSTRUCCIÓN DE LA BASE DE DATOS}

La base de datos consta de un conjunto de imágenes digitales de la superficie de los proyectiles 9 mm disparados por diferentes tipos de armas: dos sub-ametralladoras MP5, una sub-ametralladora UZI y una Glock modelo 17 (A, B, C, D respectivamente en las tablas de datos). Esta información es relevante, debido a que el tipo de estriado que deja este conjunto de armas es poligonal, lo cual representa una dificultad en la descripción de las imágenes por la irregularidad de las marcas que quedan sobre las superficies de los proyectiles.

De los proyectiles disparados por cada arma, fueron extraídos aleatoriamente cuatro de ellos para formar la base de datos. Las imágenes representan la superficie "desenvuelta” de la zona de interés que contiene las marcas generadas al contacto con el interior del cañón durante el disparo.

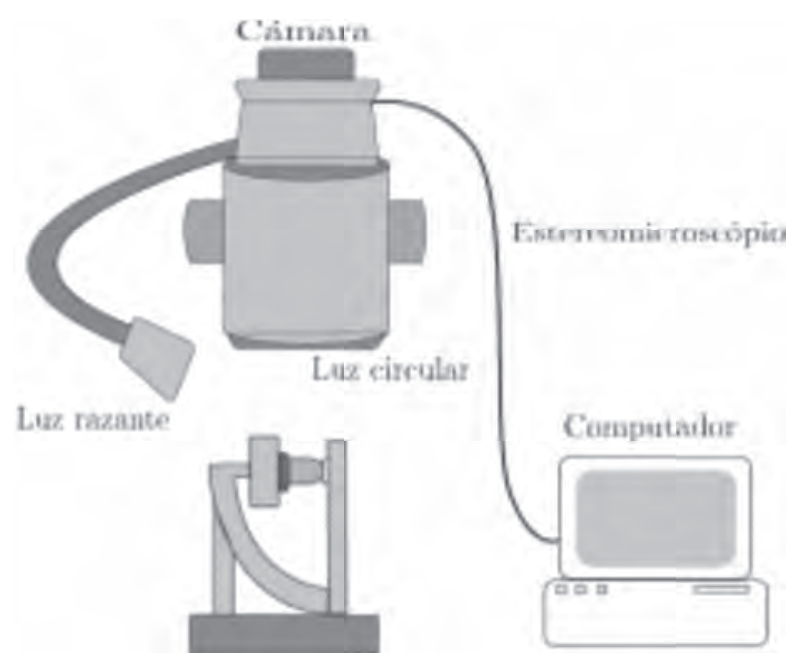

Figura 1. Disposición de equipos para la adquisición de las imágenes 
La disposición de equipos para la adquisición de imágenes (Figura 1), es un sistema fotográfico digital de alto rendimiento para fotomicrografía de alta resolución (Leica DFC490), microscopio estereoscópico Leica MZ6 y Software LAS (Leica Application Suite). El procedimiento para obtener imágenes "desenvueltas" de los proyectiles, consiste en tomar una fotografía del proyectil cada $12^{\circ}$ (Figura 2.a), segmentar la sección de interés de cada una de las que contienen estrías y los macizos, luego concatenar los segmentos, usando interpolación lineal entre las intensidades presentes en las fronteras, hasta formar las imágenes de la superficie completa (Figura 2.b).

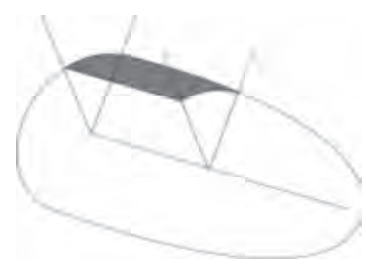

a)

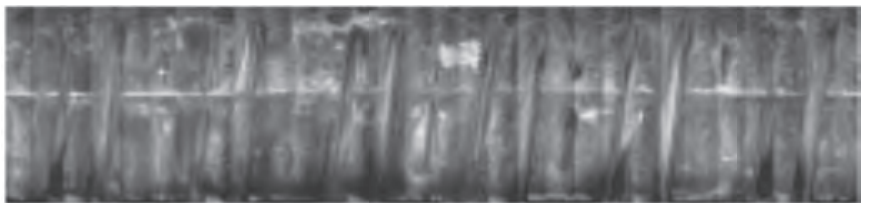

b)

Figura 2. (a) Segmento extraído de cada fotografía (b) Superficie “desenvuelta" del proyectil

La razón para considerar que las rotaciones de $12^{\circ}$ son suficientes para tomar como constante la intensidad de la luz en cada segmento, es que la diferencia de alturas entre los extremos y la parte central es de $0.300 \mathrm{~mm} \pm 0.026 \mathrm{~mm}$.

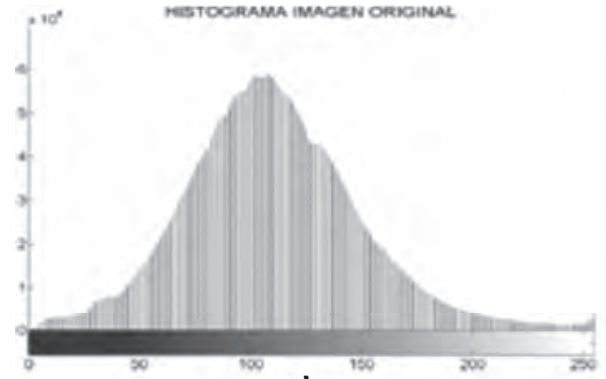

a)

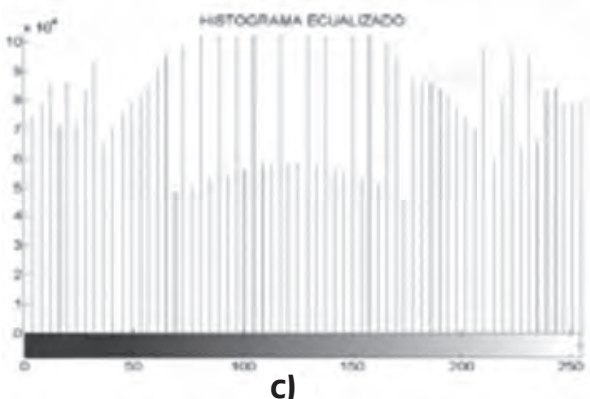

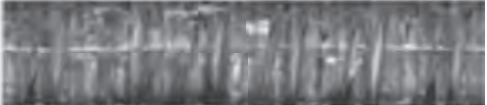

b)

Figura 3. a) Histograma de la superficie original, b) Imagen original, c) Histograma ecualizado, d) Imagen de mayor contraste obtenida usando la ecualización del histograma (a) 
Fue necesaria una mejora de contraste entre las imágenes, debido a que usualmente se obtienen imágenes borrosas por la fuerte reflexión en la superficie del material [3], o por otros factores como: inadecuado y/o pobre entorno de iluminación, deficiencia en el rango dinámico del sensor o apertura errónea de las lentes durante la adquisición de la imagen [4].

Las imágenes incluidas en este trabajo, presentaron bajo contraste caracterizado por una alta concentración en la imagen de niveles de gris con valores intermedios (Figuras 3.a y 3.b). La técnica usada en la mejora del contraste de las imágenes en el presente trabajo, fue la ecualización del histograma [5]. Esta técnica permite ampliar el rango dinámico de los niveles de gris presentes en la imagen, proporcionando una aproximación a un histograma plano (Figuras 3.c y 3.d).

\subsection{ESPACIOS CARACTERÍSTICOS}

Para describir las imágenes se construyeron tres espacios característicos que se usaron para probar la significancia estadística de las diferencias entre grupos de proyectiles provenientes de las armas. Los enfoques usados para construir los espacios característicos fueron: dos enfoques estadísticos, uno de ellos basado en el histograma de las imágenes y el otro en la Matriz de co-ocurrencia de niveles de gris (MCNG); y un enfoque espectral, basado en la distribución de las intensidades de niveles de gris, usando las frecuencias espaciales de los espectros de Fourier obtenidos a partir de las imágenes.

Teniendo en cuenta que una imagen se puede tratar como un conjunto discreto de datos organizado en una matriz $\mathrm{M}$ x N, donde cada dato representa la intensidad de determinado píxel, el histograma de la imagen representa la probabilidad de que un píxel tenga determinado valor. El $n$-ésimo momento cercano a la media de un conjunto discreto de datos está dado por $[4,5]$ :

$$
\mu_{n}=\sum_{i=0}^{L}\left(z_{i}-m\right)^{n} p\left(z_{i}\right)
$$

Donde $z_{i}$ representa una variable aleatoria discreta, $p(z)$ representa el histograma de la imagen, $L$ es el máximo nivel de gris que puede tomar la imagen (depende si la imagen es de 8- bits, 16-bits, etc.), y $m$ es la media o intensidad promedio de la imagen.

A continuación se presenta un conjunto de descriptores útiles en la representación estadística de las características de las imágenes digitales [4, 5].

$$
m=\sum_{i=0}^{L} z_{i} p\left(z_{i}\right) \text { Intensidad promedio }
$$




$$
\begin{aligned}
\sigma & =\sqrt{m_{2}(z)} \quad \text { Contraste promedio } \\
R & =1-\frac{1}{1+\sigma^{2}} \quad \text { Suavidad relativa } \\
\mu_{3} & =\sum_{i=0}^{L}\left(z_{i}-m\right)^{3} p\left(z_{i}\right) \text { Asimetría del histograma } \\
U & =\sum_{i=0}^{L} p\left(z_{i}\right)^{2} \quad \text { Uniformidad en niveles de gris } \\
e & =-\sum_{i=0}^{L} p\left(z_{i}\right) \log p\left(z_{i}\right) \text { Entropía }
\end{aligned}
$$

\section{Matriz de co-ocurrencia de niveles de gris (MCNG) [4]}

Definición 1. Sea $\mathbf{P}$ un operador de posición y $\mathbf{A}$ una matriz $k \times k \mid k$ : número de niveles de gris diferentes en la imagen). El elemento $a_{i j}$ representa el número de veces que aparecen los puntos con el nivel de gris $z_{i}$, en la posición especificada por $\boldsymbol{P}$, en relación con los puntos con un nivel de gris $z_{j}$. Con $i \geq j$. Luego la MCNG, puede ser calculada a partir de:

$$
\boldsymbol{C}=\frac{1}{n} \boldsymbol{A}
$$

Donde $n$ ( $n=\sum a_{-} i j$ ), es el número total de puntos que satisfacen el operador de posición $\mathbf{P}$ (como el usado en el presente trabajo: primer vecino a la derecha). Por su parte, $c_{i j}$ representa entonces, la probabilidad conjunta de que un par de puntos que satisfacen $\mathbf{P}$ tengan valores $\left(z_{i}, z_{j}\right)$. De hecho, eligiendo un adecuado operador de posición, se puede determinar un patrón en una textura dada. Un conjunto de descriptores útil en la tarea de categorizar una textura, usando dicha matriz, según [4] es:

$$
\begin{gathered}
\sum_{i} \sum_{j}|i-j|^{2} c_{i j} \text { Contraste } \\
\sum_{i} \sum_{j} \frac{\left(i-\mu_{i}\right)\left(j-\mu_{j}\right) c_{i j}}{\sigma_{i} \sigma_{j}} \quad \text { Correlación }
\end{gathered}
$$




$$
\begin{gathered}
\sum_{i} \sum_{j} c_{i j}{ }^{2} \text { Energía o uniformidad } \\
\sum_{i} \sum_{j} \frac{c_{i j}}{1+|i-j|} \text { Homogeneidad } \\
\sum_{i} \sum_{j} c_{i j} \log \left(c_{i j}\right) \quad \text { Entropía }
\end{gathered}
$$

Por último, se usó el espectro de Fourier de cada imagen para efectos de la descripción, porque permite detectar fácilmente la presencia de patrones periódicos o cuasi-periódicos globales en las imágenes.Tales patrones, aunque son fáciles de distinguir como concentraciones de alta energía en el espectro, son muy difíciles de detectar, usando métodos de dominio espacial por la naturaleza local de estas técnicas [2]. La transformada de Fourier de una función continua de dos variables $f(x, y)$, está definida por:

$$
F(u, v)=\int_{-\infty}^{\infty} f(x, y) e^{-j 2 \pi(u x+v y)} d x d y
$$

Donde: $j=\sqrt{-1}$

La transformada de Fourier de una función discreta de dos variables (imagen digitalizada), $f(x, y)$, de tamaño $\mathrm{M} \times \mathrm{N}$, está dada por la ecuación:

$$
F(u, v)=\frac{1}{M N} \sum_{x=0}^{M-1} \sum_{y=0}^{N-1} f(x, y) e^{-j 2 \pi\left(\frac{u x}{M}+\frac{v y}{N}\right)}
$$

Donde: $u=0,1,2, \ldots, M-1 ; v=0,1,2, \ldots, N-1$.

En el presente trabajo fueron consideradas tres características del espectro de Fourier que son útiles para describir la textura [5]:

- Picos prominentes del espectro que dan la dirección principal de los patrones de textura

- La localización de los picos en el plano de la frecuencia que proporciona el período espacial fundamental de los patrones.

- La eliminación de componentes periódicos mediante el filtrado deja elementos no periódicos de la imagen, que se pueden describir por medio de técnicas estadísticas.

La detección e interpretación de las características es simplificada y expresa el espectro en coordenadas polares para producir una función $S(r, \theta)$, siendo $S$ la función del espectro y $(r, \theta)$ las variables de este sistema de coordenadas. Para cada dirección $\theta$, se puede considerar $S(r, \theta)$ como una función unidimensional; de forma similar para cada frecuencia $r, S(r, \theta)$ se puede considerar 
como unidimensional $S_{r}(\theta)$. Analizando $S_{\theta}(r)$, para un valor fijo de $\theta$, se obtiene el comportamiento del espectro (tal como la presencia de picos) a lo largo de una dirección radial desde el origen; mientras que analizando $S_{r}(\theta)$ para un valor fijo de $r$, se obtiene el comportamiento a lo largo de un semicírculo de radio $r$ centrado en el origen. Luego, una descripción más global se puede obtener, integrando (sumando variables discretas), así:

$$
\begin{aligned}
& S(r)=\sum_{\theta=0}^{\pi} S_{\theta}(r) \\
& S(\theta)=\sum_{r=1}^{R} S_{r}(\theta)
\end{aligned}
$$

Para un espectro $\mathrm{N} \times \mathrm{N}$, se escoge $S=N / 2$; en general, para un espectro $\mathrm{M} \times \mathrm{N}$ se toma $R=$ mayor $(M, N) / 2$.

Los resultados de las ecuaciones 16 y 17 constituyen un par de valores $[S(r), S(\theta)$ ] para cada par de coordenadas $(r, \theta)$. Variando estas coordenadas, se puede obtener dos funciones unidimensionales, $S(r)$ y $S(\theta)$ que constituyen una descripción de la energía espectral de la textura para una región o para una imagen completa. Más aún, los mismos descriptores de estas funciones se pueden calcular para caracterizar su comportamiento cuantitativo.

Un conjunto de valores útil, según $[3,4,5]$, para la descripción de la energía espectral de la textura a partir de las funciones unidimensionales $S(r)$ y $S(\theta)$ incluye:

- Localización del valor máximo

- La media y la desviación estándar

- Posición del máximo de la función angular $(S(\theta))$

- Relación entre el valor máximo y el medio

Este último descriptor no se incluye en el modelo lineal general multivariante del análisis de la varianza, debido a que es una combinación lineal de otros dos descriptores incluidos en el análisis. Esta condición de las matrices de datos, hace que la misma sea Definida No Positiva y por lo tanto, fallan los intentos de realizar la prueba de significancia estadística de las diferencias entre los vectores de medias, como se muestra más adelante en el análisis de datos.

\section{RESULTADOS}

Una vez construidas las matrices que contenían las características de las imágenes de diferentes armas, calculadas usando los enfoques ya descritos, se usaron para hacer el análisis de la significancia estadística de sus diferencias. 


\subsection{ANÁLISIS UNIVARIADO DE LA VARIANZA: ANOVA}

Se determinó si la media de las características evaluadas difería entre los proyectiles provenientes de diferentes armas de fuego (se calcularon intervalos de confianza de 95\%). ANOVA consiste en comparar la variabilidad al interior de cada grupo con la variabilidad real entre los grupos; para contrastar la hipótesis nula en la cual todas las medias de las diferentes poblaciones son iguales, contra la hipótesis alternativa en la cual al menos, dos de las medias grupales son diferentes. Vale mencionar que para el presente trabajo, cada grupo o nivel representa un arma diferente.

Antes de realizar el análisis de varianza, se hicieron las pruebas respectivas de normalidad de los datos de cada grupo, y homogeneidad de las varianzas de los grupos, con el fin de verificar los supuestos teóricos subyacentes a esta técnica. Como resultado, las pruebas de Leveney de Shapiro-Wilk respectivamente, indicaron que efectivamente los datos de cada grupo provienen de poblaciones normales, y que las varianzas son homogéneas; lo cual fue verificado para cada característica o variable.

Tabla 1. Sumario de grupos diferentes bajo ANOVA

\begin{tabular}{|c|c|c|c|}
\hline NATURALEZA DE LAS CARACTERÍSTICAS & CARACTERÍSTICAS & GR & RENTES \\
\hline \multirow{12}{*}{ MCNG } & \multirow{3}{*}{ Contraste } & $A$ & $B$ \\
\hline & & $\mathrm{B}$ & C \\
\hline & & $B$ & $\mathrm{D}$ \\
\hline & \multirow{2}{*}{ Correlación } & $B$ & C \\
\hline & & $B$ & $\mathrm{D}$ \\
\hline & \multirow{3}{*}{ Energía } & A & B \\
\hline & & $B$ & C \\
\hline & & $\mathrm{B}$ & $\mathrm{D}$ \\
\hline & \multirow{4}{*}{ Homogeneidad } & A & $\mathrm{B}$ \\
\hline & & A & $\mathrm{D}$ \\
\hline & & $\mathrm{B}$ & C \\
\hline & & $B$ & D \\
\hline \multirow{3}{*}{ Histograma } & \multirow{2}{*}{ Uniformidad } & $\mathrm{B}$ & C \\
\hline & & $B$ & $\mathrm{D}$ \\
\hline & Entropía & B & $\mathrm{D}$ \\
\hline \multirow{5}{*}{ Espectro de la imagen } & \multirow{2}{*}{ Desviación estándar S( r ) } & A & C \\
\hline & & $\mathrm{B}$ & C \\
\hline & \multirow{3}{*}{ Posición del Máximo $S(\mu)$} & A & B \\
\hline & & B & C \\
\hline & & $B$ & $D$ \\
\hline
\end{tabular}

Fuente: Méndez García Jeison, 2010 [8]

La técnica de múltiples comparaciones de medias [6], se usó para determinar los casos en los cuales se rechazó la hipótesis nula (igualdad de medias entre los grupos de proyectiles de diferentes armas de fuego), y cuáles de los grupos tenían medias diferentes. Los resultados se muestran en la Tabla 1. 
En la Tabla 1, se encuentran organizados los grupos de proyectiles que se diferencian estadísticamente. Por ejemplo: al inicio de la tabla se puede observar que el grupo de proyectiles identificados con el código B (proyectiles $9 \mathrm{~mm}$ de una sub-ametralladora UZI), se diferencian estadísiticamente de los otros grupos de proyectiles con respecto del contraste calculado a partir de las matrices de co-ocurrencia de niveles de gris. Usando las comparaciones múltiples en el caso univariado, se observa que el grupo de proyectiles que más se diferencia del resto, es el grupo B (proyectiles 9 mm de una sub-ametralladora MP5). Sin embargo, no se presenta discriminación considerable para los otros conjuntos de proyectiles.

\subsection{ANÁLISIS MULTIVARIADO DE LA VARIANZA: MANOVA}

MANOVA es una técnica de dependencia que mide las diferencias de dos o más variables métricas dependientes basadas en un conjunto de variables independientes categóricas (no métricas) para efectos de predicción. El MANOVA es proceso multivariado que se usa para valorar la diferencia entre grupos por medio de múltiples variables de manera simultánea [6].

En esta prueba, se contrastó la hipótesis nula de igualdad de vectores multivariados de medias de las muestras poblacionales, para las características medidas; con la hipótesis alternativa en la cual al menos, dos de estos vectores de medias multivariadas son diferentes.

Para realizar esta prueba, se hicieron antes las pruebas sobre los supuestos de correlación de las variables y de homocedasticidad del conjunto de datos: homogeneidad de las matrices de varianzas-covarianzas.

El resultado de la prueba de Esfericidad de Bartlett, que permite contrastar la hipótesis nula que la matriz de correlaciones de las variables dependientes se asemeja a una matriz identidad (que no existe correlación entre las variables), muestra que el nivel de significancia (p-valor), para cada conjunto de datos es menor al 5\%, lo cual es evidencia necesaria y suficiente para concluir que al menos para un intervalo de confidencia del 95\%, se puede rechazar la hipótesis nula en la cual las variables no tienen correlación alguna.

La prueba de $M$ de Boxno se realizó, dado que sólo se tenían cuatro observaciones para cada tipo de arma en las diferentes variables. La prueba arriba mencionada tiene como supuesto teórico que el tamaño de la muestra sea mayor al número de variables independientes [6].

En el caso de diseños balanceados (como en el presente trabajo), donde todos los grupos tienen un tamaño muestra igual, los cuatro estadísticos usados para probar la hipótesis nula de igualdad de los vectores multivariados de medias: Lambda de Wilks, Traza deLawley-Hotelling, Traza de Pillai-Bartlett y Raiz más grande de Roy, son lo suficiente fuertes para manejar, en el caso de que se presente, los conjuntos de datos que muestren heterogeneidad en sus matrices de varianzas-covarianzas [7].

En la Tabla 2, se muestran los resultados de la prueba MANOVA. Para encontrar el nivel de significancia de la prueba, se usaron aproximaciones a la distribución F con grados de libertad: gl. Hip y gl. Error. 
Tabla 2. Sumario de resultados bajo MANOVA

\begin{tabular}{|l|l|r|r|c|}
\hline & Estadistico & Valor & (gl. Hip, gl. Error) & p-valor \\
\hline \multirow{5}{*}{ MCNG } & Traza de Pillai & 1.9025 & $(12,33)$ & 0.0002 \\
& Lambda de Wilks & 0.0218 & $(12,24.1030)$ & 0.0001 \\
& Traza de Hotelling & 10.2595 & $(12,23)$ & 0.0001 \\
& Raiz más grande de Roy & 5.9967 & $(4,11)$ & 0.0001 \\
\hline \multirow{5}{*}{ Histograma } & Traza de Pillai & 1.9321 & $(18,27)$ & 0.0093 \\
& Lambda de Wilks & 0.0374 & $(18,20.2840)$ & 0.0258 \\
& Traza de Hotelling & 6.5095 & $(18,17)$ & 0.0729 \\
& Raiz más grande de Roy & 3.4451 & $(6,9)$ & 0.0145 \\
\hline \multirow{5}{*}{$S(r)$} & Traza de Pillai & 1.9321 & $(18,27)$ & 0.0093 \\
& Lambda de Wilks & 0.0374 & $(18,20.2840)$ & 0.0258 \\
& Traza de Hotelling & 6.5095 & $(18,17)$ & 0.0729 \\
& Raiz más grande de Roy & 3.4451 & $(6,9)$ & 0.0145 \\
\hline \multirow{5}{*}{$S(\theta)$} & Traza de Pillai & 1.5979 & $(12,33)$ & 0.0046 \\
& Lambda de Wilks & 0.0461 & $(12,24.1030)$ & 0.0010 \\
& Traza de Hotelling & 8.9182 & $(12,23)$ & 0.0002 \\
& Raiz más grande de Roy & 21.2222 & $(4,11)$ & 0.0000 \\
\hline
\end{tabular}

En la Tabla 2, se puede observar que los estadísticos muestran que los vectores multivariados de medias presentan diferencias estadísticas significativas, salvo en dos casos (Traza de Lawley-Hotelling para las características del histograma y la parte radial del espectro de la imagen). Así mismo, los p-valormás bajos se hallan para las características de la MCNG, y confirman que a partir de estas se obtienen valores que discriminan mejor las diferencias entre las texturas caracterizadas.

\subsection{ANÁLISIS DE DISCRIMINACIÓN LINEAL}

Para llevar a cabo el análisis discriminante, usando el modelo Lineal de Fisher, se tuvo en cuenta las variables canónicas: "supervariables" que contienen la información de todas las variables del conjunto de datos original, obtenidas a partir de la relación que se muestra a continuación:

$$
(X-m)_{\mathrm{N} \times p} * V_{p \times p}=C \mathrm{~N} \times p
$$

Donde cada $x_{i}$ representa un vector de dimensión $p$ que contiene las observaciones para cada elemento de los $k n=N$ ( $k$ grupos y $n$ el tamaño muestral de cada uno), $\boldsymbol{m}$ es un vector $p$-dimensional que contiene en cada elemento el valor medio de las observaciones para cada variable y $\boldsymbol{V}$ es la matriz que contiene los $p$ vectores propios de $\boldsymbol{W}^{-\mathbf{1}} \boldsymbol{B}$ ( $\boldsymbol{W}$ : varianza-covarianza intra-grupo y, B: varianza-covarianza entre-grupos), rescatados de forma tal que la varianza intragrupos de las variables canónicas contenidas en $\boldsymbol{C}$ es igual a la matriz identidad. 


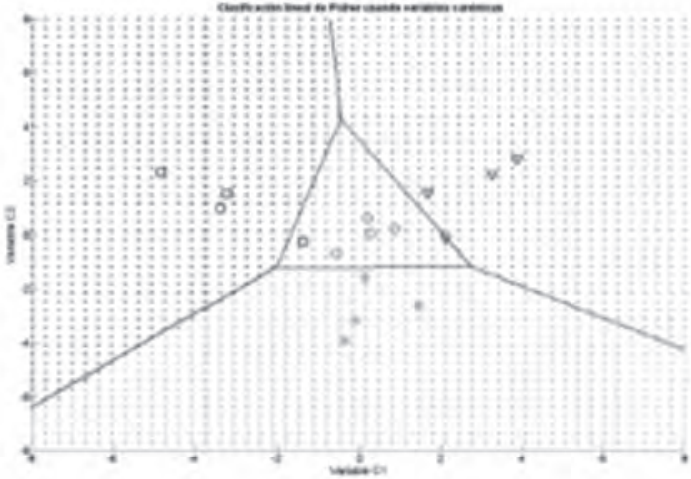

(a)

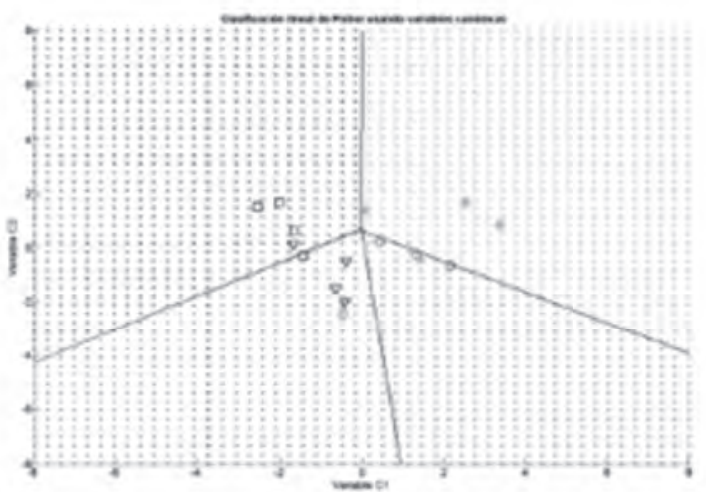

(c)

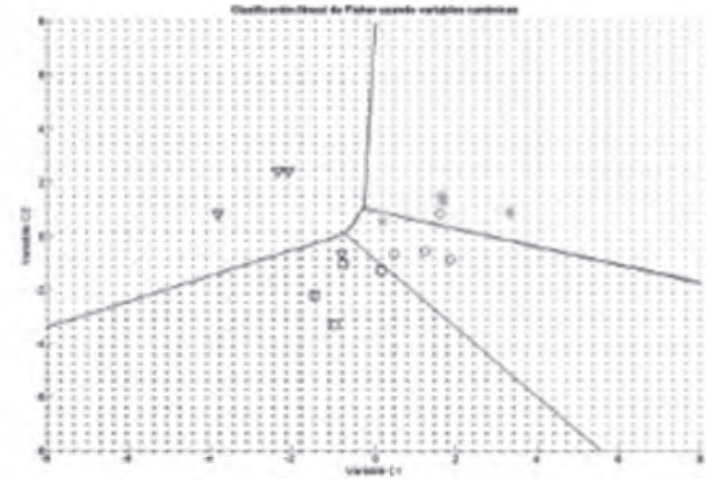

(b)

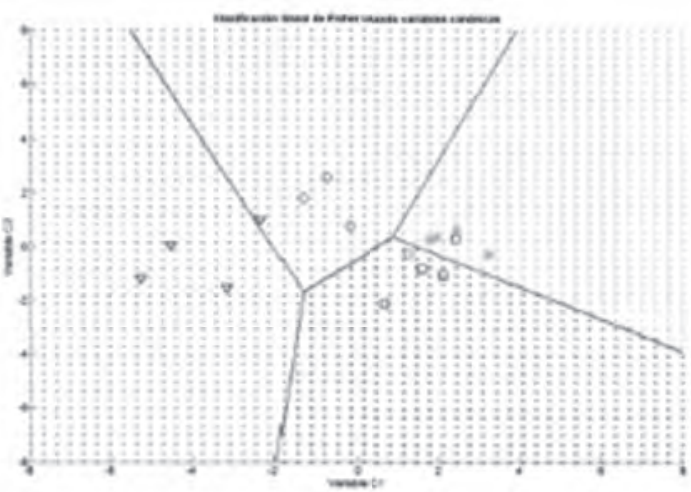

(d)

Figura 4. Análisis discriminante a) MCNG, b) Histograma,

c) y d) funciones espectrales radial y angular.

Fuente: Méndez García Jeison 2010 [8]

En la Figura 4, se muestra el resultado del análisis discriminante lineal de Fisher a las variables canónicas. Se muestra cómo están distribuidos en este espacio, cada grupo de proyectiles (A: rojo, B: azul, C: negro y D: verde), y a qué grupo sería asignado un elemento nuevo, dependiendo de la región donde se encuentre ubicado. Para la construcción de cada gráfico, se usaron los dos primeros conjuntos de variables canónicas calculados a partir de las diferentes matrices de observaciones. Se encontraron regiones mejor definidas para los gráficos a) y b), lo cual indica una mayor discriminación en el caso multivariado. 


\section{CONCLUSIONES}

En esta investigación, a partir del proyectil físico disparado por un arma de fuego, se logró la representación bidimensional de su superficie mediante rotaciones de $12^{\circ}$. Las imágenes obtenidas al concatenar los segmentos de interés, muestran una continuidad en la imagen, lo cual permite concluir que el proceso de “desenvolvimiento" de la superficie del proyectil fue satisfactorio.

Las pruebas de significación estadística de las diferencias entre los proyectiles provenientes de diferentes armas: ANOVA y MANOVA, permiten concluir para un nivel de significación del 95\%, que existían diferencias entre las marcas dejadas sobre la superficie de los proyectiles disparados por las armas de fuego estudiadas en esta investigación. Adicionalmente, las características extraídas de la matriz de co-ocurrencia de niveles de gris (MCNG), a partir de las imágenes de los proyectiles, permitieron distinguir mejor las marcas dejadas sobre la superficie de los proyectiles de las diferentes armas, que las características obtenidas a partir de los histogramas y los espectros de las imágenes de las superficies de los mismos proyectiles.

Como futura extensión del presente trabajo, quedo planteado el estudio y aplicación de técnicas de compresión de imágenes que permitan reducir el costo computacional. Además, se propuso segmentar las regiones de la superficie de los proyectiles en donde se encuentran las estrías, con el objeto de comparar los resultados con los obtenidos en la presente investigación, en la cual se hizo un estudio, contemplando toda la superficie.

\section{AGRADECIMIENTOS}

Cordial agradecimiento al Laboratorio de comparación balística de la Fiscalía de Pereira, en especial al Doctor Jaime Granada Hincapié. Además, el proyecto fue parcialmente financiado por la Vicerrectoría de Investigaciones Innovación y Extensión de la Universidad Tecnológica de Pereira, bajo la VI convocatoria interna para financiar trabajos de grado.

\section{REFERENCIAS BIBLIOGRÁFICAS}

[1] Bonfanti M.S., y De Kinder J., (1999). The influence of manufacturing processes on the identification of bullets and cartridge cases - a review of literature. En: Science and Justice, 39(1), pp. 3-10.

[2] Dongguang Li, (2008). Firearm identification system based on ballistics imageprocessing. Congress on Image and Signal Processing (CISP 2008), Hainan, China, pp. 149-154.

[3] Dongguang Li, (2006). Ballistics projectile image analysis for firearm identification. En: IEEE Transactions on Image Processing, Vol. 15 (10), pp. 2857-2865. 
[4] González R.C., y Woods R.E., (1992). Tratamiento digital de imágenes. Addison-Wesley, Inc. Delaware.

[5] González R.C., and Woods R.E., (2002). Digital image processing using Matlab. AddisonWesley, Inc. Delaware.

[6] Hair J.F., y Anderson R.E., (1999). Análisis multivariante. Madrid. Prentice Hall, Madrid.

[7] Rencher A.C., (2002). Methods of multivariate analysis. Jonh Wiley \& Sons, New York.

[8] Diseño de la metodología para caracterizar texturas presentes en imágenes de proyectiles disparados por armas de fuego, con el fin de realizar cotejo automático. En: http:// recursosbiblioteca.utp.edu.co/tesisdigitales/ficha814.htmL. (junio de 2010). 
